\title{
星载多型号光电探测器热真空环境试验研究
}

陈 志 ${ }^{1,2}$, 汪杰君 ${ }^{3}$, 胡亚东 ${ }^{2}$, 张爱文 ${ }^{2}$, 厉卓然 ${ }^{2}$, 崔珊珊 ${ }^{2}$, 金 洁 $^{2}$, 洪 津 $^{2}$ (1. 桂林电子科技大学电子工程与自动化学院, 广西 桂林 541004; 2. 中国科学院安徽光学精密机械研究所, 安徽 合肥 230031; 3. 桂林电子科技大学北海校区海洋工程学院, 广西 北海 536000)

\begin{abstract}
摘要: 为将低等级探测器应用于宇航等高可靠性应用环境, 对探测器进行可靠性环境试验考核至关重 要。本文提出了一种探测器的热真空实验方法, 对多种型号星载探测器进行热真空环境考核试验, 通 过对比热真空环境试验前后探测器的相对光谱响应率、暗电流、制冷器驱动电流、探测器制冷特性等 参数的变化, 分析各型号探测器热真空环境的适应性, 从而在早期暴露可能存在质量及其他缺陷的产 品, 篎选出性能最优产品用于宇航产品。试验结果表明, 参试的探测器在热真空环境试验考核和篮选 之后的各项性能指标满足设计要求, 具有很好的可靠性, 可以满足航天载荷应用需求。
\end{abstract}

关键字: 航天载荷; 探测器; 可靠性; 热真空试验; 篎选

中图分类号：TP732 文献标志码：A 文章编号：1001-8891(2020)09-0823-06

\section{Thermal Vacuum Environment Tests of Multimodel Photodetectors on Satellites}

\author{
CHEN Zhi ${ }^{1,2}$, WANG Jiejun ${ }^{3}$, HU Yadong ${ }^{2}$, ZHANG Aiwen ${ }^{2}$,
}

LI Zhuoran ${ }^{2}$, CUI Shanshan ${ }^{2}$, JIN Jie ${ }^{2}$, HONG Jin $^{2}$

(1. College of electronic engineering and automation, Guilin University of Electronic Science and technology, Guilin 541004, China;

2. Anhui Institute of Optics and precision machinery, Chinese Academy of Sciences, Hefei 230031, China;

3. College of marine engineering, Beihai campus, Guilin University of Electronic Science and Technology, Beihai 536000, China)

\begin{abstract}
To apply low-level detectors in high-reliability application environments, such as those in the aerospace field, it is important to test the environmental reliability of such detectors. In this paper, a thermal vacuum experiment method is proposed to test various types of space-borne detectors. By comparing the changes in the relative spectral response rate, dark current, driving current of the refrigerator, and refrigeration characteristics of each type of detector before and after the thermal vacuum environment test, their adaptabilities in the thermal vacuum environment are analyzed to enable early popularization. To avoid products with possible quality and other defects, only those products demonstrating the best performances are selected for aerospace applications. The results show that based on the performance indices of detectors that meet the design requirements after the thermal vacuum environment testing and screening, such devices have good reliability and stability, which can meet the requirements of aerospace loads.
\end{abstract}

Key words: aerospace load, detector, reliability, thermal vacuum test, screening

\section{0 引言}

探测器关键性能参数主要包括暗电流、相对光谱 响应度、制冷特性等 ${ }^{[1]}$, 暗电流作为探测器的重要性 能指标, 是探测系统性能的最重要影响因素, 具有较 强的温度敏感性, 温度波动将直接影响系统暗电流变 化从而影响短波红外偏振测量仪器测量精度 ${ }^{[2-3]}$ 。此 外, 探测器的响应度和制冷特性等, 也是探测系统性
能的重要影响因素。

为了满足不同型号低等级探测器在高精度高可 靠性领域的应用需求, 文献[4-5]提出一种方法将温变 速率这一应力作为主要研究对象, 对多种型号同批次 星载红外探测器进行加速寿命试验, 并对试验前后探 测器相对光谱响应率和制冷器驱动电流进行分析。文 献[6]提出对星载红外探测器组件进行温度循环试验、 力学试验以及高温老炼试验等环境试验考核, 通过对 
比环境试验前后红外探测器组件相对光谱响应率变 化, 分析红外探测器组件的环境适应性。文献[7]提出 了一种有利于空间外差光谱仪探测器的篮选方法, 利 用设计的探测器篮选装置进行了探测器篮选实验, 对 实验数据进行处理和分析, 从备选探测器中选取了最 优性能的探测器。文献[8]介绍了 CCD 的篮选流程, 第一步是从各 CCD 裸片的信噪比、相对光谱响应、 暗电流等角度进行篎选, 将不满足指标的 CCD 剔除。

本文设计并提出了一种探测器的热真空实验方 法, 在文献[6]的基础上对多种型号的星载探测器组件 进行进一步的环境考核试验, 并对环境试验前后探测 器的相对光谱响应率、暗电流、制冷器驱动电流、探 测器制冷特性等性能进行测试。通过对比热真空环境 试验前后探测器各性能的变化, 分析各型号探测器热 真空环境的适应性, 从中优选出具有高可靠性高稳定 性的探测器进行高精度大气偏振探测应用。

\section{1 光电探测器失效模式分析}

实践证明大多数设备的故障率是时间的函数, 典 型故障曲线称之为浴盆曲线（Bathtub curve），如图 1 所示。浴盆曲线具有明显的阶段性, 失效率随使用 时间变化分为 3 个阶段: 早期失效期（I ）、偶然失 效期（II）和耗损失效期（III） ${ }^{[6,9]}$ 。

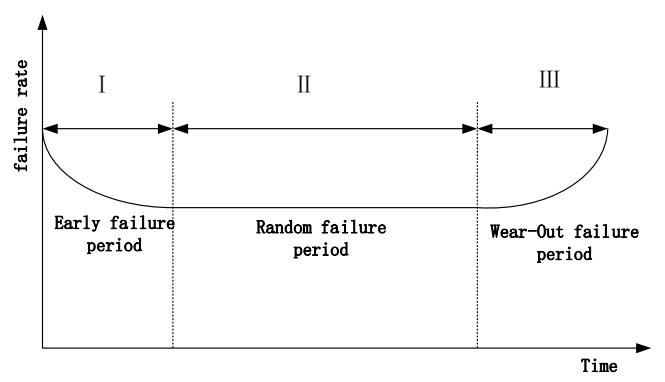

图 1 浴盆曲线

Fig.1 Bathtub curve

在 I 以前是早期失效阶段, 主要由制造、装配、 质量控制与检验不严引起的, 中间为平坦的随机失效 阶段, II 以后为老化阶段, 起始与末尾期失效率很高, 这说明在起始期要严格篎选 ${ }^{[9]}$ 。

\section{2 热真空试验}

本文所述的多型号星载探测器主要包括 5 只硅探 测器 S13735、9 只红外探测器（5 只 InGaAs G12180 探测器以及 4 只 InGaAs G12183 探测器），其中硅探 测器典型响应波长分别为 $490 \mathrm{~nm} 、 550 \mathrm{~nm} 、 670 \mathrm{~nm}$ 、 $870 \mathrm{~nm} 、 910 \mathrm{~nm}$, InGaAs G12180 红外探测器的典型 响应波长为 $1380 \mathrm{~nm}$ 和 $1610 \mathrm{~nm}, \mathrm{G} 12183$ 型红外探测 器的典型响应波长为 $2250 \mathrm{~nm}$ 。这些型号的探测器在
出厂前已由厂家对其进行了一些可靠性试验, 本文的 主要目的是对这些型号的探测器进行进一步的热真 空环境适应性分析从而实现二次笁选, 确保探测器在 实际使用时能够安全可靠地工作。

参考探测器使用说明并结合探测器实际运行环 境条件, 设置了探测器热真空环境试验条件, 在保证 环境试验条件对探测器的篮选力度的同时防止环境 试验应力过大对探测器产生过试验而损伤器件。

\section{1 试验条件}

探测器的热真空试验主要是模拟真实在轨运行 环境的温度、真空环境、极端高低温以及主要温度工 作点等环境条件进行循环试验, 考核其工作的稳定性 和可靠性。设计热真空实验条件如表 1 所示。

\section{表 1 热真空试验条件}

Table 1 Thermal vacuum test conditions

\begin{tabular}{ccc}
\hline Temperature & $\begin{array}{c}\text { Temperature holding } \\
\text { time }\end{array}$ & $\begin{array}{c}\text { Performance test } \\
\text { holding time }\end{array}$ \\
\hline$-20^{\circ} \mathrm{C}-4^{\circ} \mathrm{C}$ & $1 \mathrm{~h}$ & 0 \\
$+55^{\circ} \mathrm{C}+4^{\circ} \mathrm{C}$ & $1 \mathrm{~h}$ & 0 \\
$-7{ }^{\circ} \mathrm{C} \pm 2{ }^{\circ} \mathrm{C}$ & 0 & $1 \mathrm{~h}$ \\
$-17^{\circ} \mathrm{C} \pm 2^{\circ} \mathrm{C}$ & 0 & $1 \mathrm{~h}$ \\
\hline
\end{tabular}

探测器热真空高低温分别为 $+55^{\circ} \mathrm{C}$ 正偏差 $4^{\circ} \mathrm{C}$ 和 $-20^{\circ} \mathrm{C}$ 负偏差 $4^{\circ} \mathrm{C}$, 性能测试温度点在 $-7^{\circ} \mathrm{C}$ 和 $-17^{\circ} \mathrm{C}$ 进行, 该温度点各进行 $1 \mathrm{~h}$ 测试, 具体的探测器热真 空循环试验状态如图 2 所示。试验温度通过真空罐制 冷、加热系统实现。

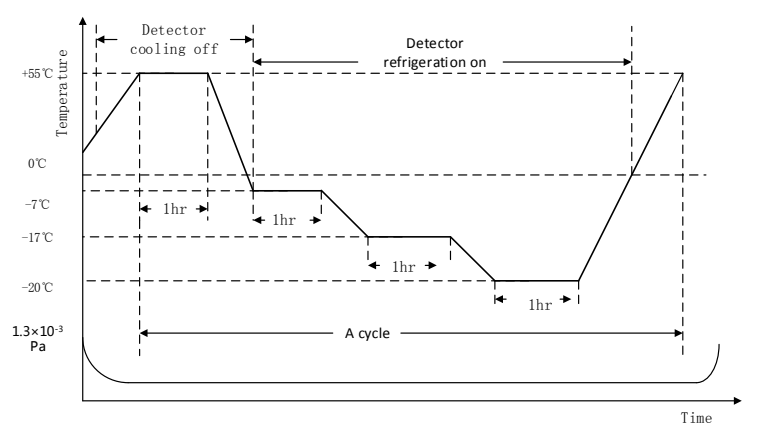

图 2 探测器热真空试验状态

Fig.2 Thermal vacuum test state of detector

\section{2 试验步骤}

1) 将测试专用设备和探测器组件按要求放置在 真空罐冷板上, 连接好地检、温度检测系统电缆, 并 检查连接状态进行系统状态确认;

2）放入防污染镜片后关闭罐门进行探测器试验 前性能测试。按照真空罐流程开始抽真空, 待真空度 优于 $1.3 \times 10^{-3} \mathrm{~Pa}$ 后, 进行工况设置;

3）设置真空罐为高温工况, 待探测器监测点温 度满足 $+55^{\circ} \mathrm{C}$ 温度点要求时, 进入热真空循环流程高 
温保持阶段;

4) 待高温保持结束后调整真空罐状态，使探测 器热沉温度为 $-7^{\circ} \mathrm{C}$, 待探测器监测点温度满足允许 的误差要求时, 开启探测器制冷器和校正仪数据采集 功能进行 $-7^{\circ} \mathrm{C}$ 性能保持测试阶段;

5） $-7^{\circ} \mathrm{C}$ 性能结束后调整真空罐状态, 使探测热 沉温度为 $-17^{\circ} \mathrm{C}$, 待监测点温度满足允许误差要求 时, 进行 $-17^{\circ} \mathrm{C}$ 性能保持测试阶段;

6) $-17^{\circ} \mathrm{C}$ 性能结束后, 设置真空罐为低温工况, 待探测器监测点温度满足 $-20^{\circ} \mathrm{C}$ 温度点要求时, 进入 热真空循环流程低温保持阶段。

按照以上流程完成 10 次近一周的循环实验, 其 真空内产品状态如图 3 所示。

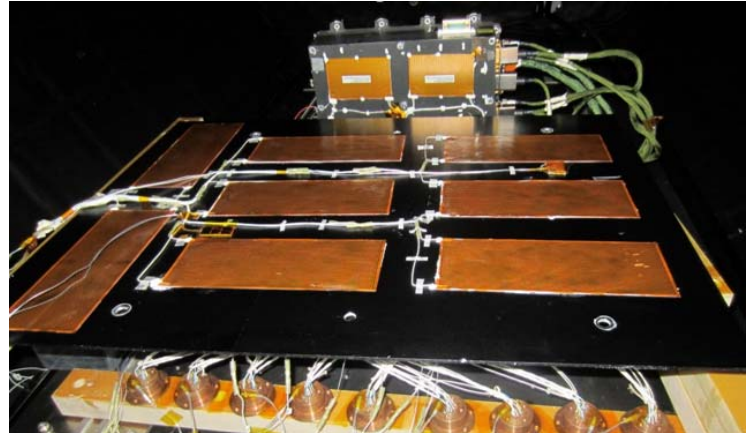

图 3 真空罐内探测器组件状态图

Fig.3 Status diagram of detector assembly in vacuum tank

\section{3 探测器试验前后各性能参数测试方法}

试验前后探测器的相对光谱响应率和暗电流的 测试是基于波段式均匀照明光源系统实现, 测试系统 如图 4 所示。积分球 $\mathrm{A}$ 内置卤铇灯作为照明光源, 产 生的光通过滤光片盒内的滤光片分光后进入积分球 $\mathrm{B}$, 积分球 $\mathrm{B}$ 匀光后进入待测探测器和参考探测器。 待测探测器与固定板连接后通过螺钉与积分球正面 中心连接, 滤光片盒内置多个与拉杆连接的滤光片拉 板, 通过调节拉杆切换不同的滤光片得到多个测试波 段的均匀光照。

利用计算机、光功率计、数字源表等配套采集设 备采集被测探测器组件相关数据。测试过程中用制冷 器驱动红外探测器组件内置 TEC (Thermo Electric Cooler）进行制冷至设定温度进行制冷驱动电流的测 试。红外探测器制冷特性的温度数据获取则是通过数 据采集器实时采集。规定波长照明下, 光电二极管探 测器相对光谱响应率计算公式见式(1):

$$
R(\lambda)=\frac{\frac{1}{m} \sum_{i=1}^{m} I(\lambda, i)-\frac{1}{m} \sum_{i=1}^{m} I_{\mathrm{dark}}(i)}{\frac{1}{n} \sum_{j=1}^{n} \Phi(\lambda, j)-\frac{1}{n} \sum_{j=1}^{n} \Phi_{\mathrm{dark}}(j)}
$$

式中： $I(\lambda, i)$ 为探测器在波长 $\lambda$ 照明下输出电流值; $I_{\mathrm{dark}}(i)$ 为探测器暗场输出电流值; $\Phi(\lambda, j)$ 为监视探头在 波长 $\lambda$ 照明下输出功率值; $\Phi_{\mathrm{dark}}(j)$ 为监视探头暗场输 出电流值; $m, n$ 为采样点个数。

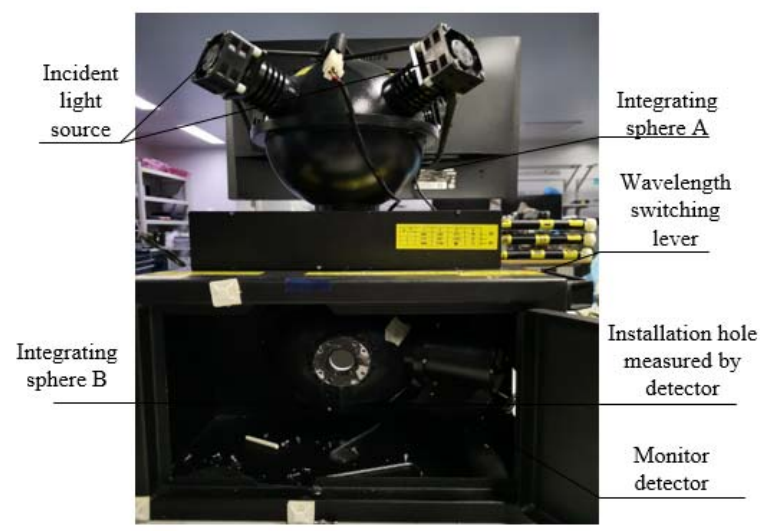

图 4 波段式均匀照明光源系统

Fig.4 Band uniform illumination light source system

试验前后各型号探测器相对光谱响应率变化率 计算公式见式(2):

$$
\Delta R(\lambda)=\frac{R\left(\lambda_{2}\right)-R\left(\lambda_{1}\right)}{R\left(\lambda_{1}\right)}
$$

式中: $R\left(\lambda_{2}\right)$ 为试验后探测器的相对光谱响应率; $R\left(\lambda_{1}\right)$ 为试验前探测器的相对光谱响应率。

\section{3 试验结果分析}

利用上述探测器环境试验装置和测试方法, 对硅 探测器 S13735、红外 InGaAs 探测 G12180 和 G12183 的 3 种型号的探测器进行热真空环境试验考核。

根据星载探测器环境试验要求, 试验前后探测器 的相对光谱响应率变化范围为 $\pm 10 \%$ 以内即满足使用 要求, S13735 和 G12180 探测器的暗电流不超过 100pA 即认为探测器性能满足需要, 对于 G12183 探 测器的试验后暗电流变化率不超过试验前 5 倍即认为 探测器性能满足需要。红外 G12180、G12183 两种型 号探测器试验前后的制冷驱动电流的变化率要求在 $\pm 10 \%$ 以内, 温控精度均满足 $\pm 0.2^{\circ} \mathrm{C}$ 的要求则探测器 制冷和温控特性满足要求。

\section{1 探测器试验前后相对光谱响应率变化}

由于探测器在实际环境工作时不需要加偏压, 所 以在测量时给探测器加 $0 \mathrm{mV}$ 偏压, 同时加 $38 \mathrm{mV}$ 偏 压的目的是为了将背景辐射降到 $1 \%$ 以下，减小温度 对探测器测量的影响。通过公式(1)计算得到 S13735、 G12180 和 G121833 种探测器的试验前后的光谱响应 率的相对变化率如图 5、图 6 所示。图中 A 和 B 分别 表示每只探测器的 A 像元和 B 像元。 


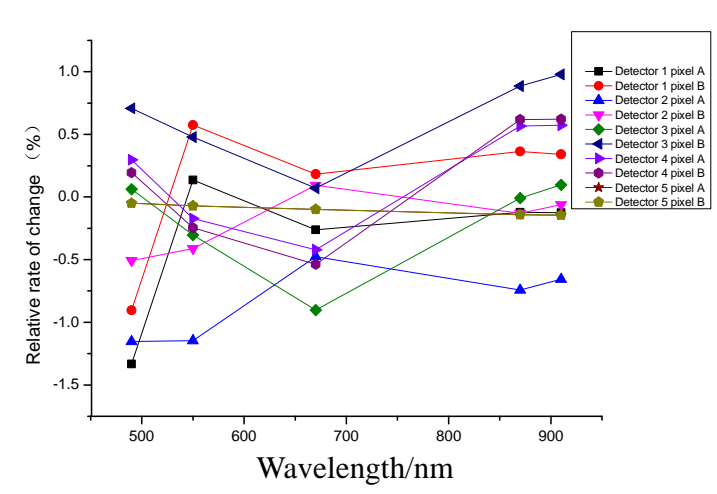

图 5 S13735 试验前后光谱响应率相对变化率

Fig.5 Relative change rate of spectral response rate before and after s13735 test

根据星载探测器环境试验要求, 从图 5、图 6 可 以看出, 各型号探测器试验前后的相对光谱响应率的 变化率都在 $\pm 3 \%$ 以内, 为了得到性能更优的探测器组 件, 根据使用数量要求, 依照优中选优的标准可以篎 选出变化率更低的探测器, 从而应用到高精度偏振和 光谱测量中。

\section{2 探测器试验前后暗电流变化}

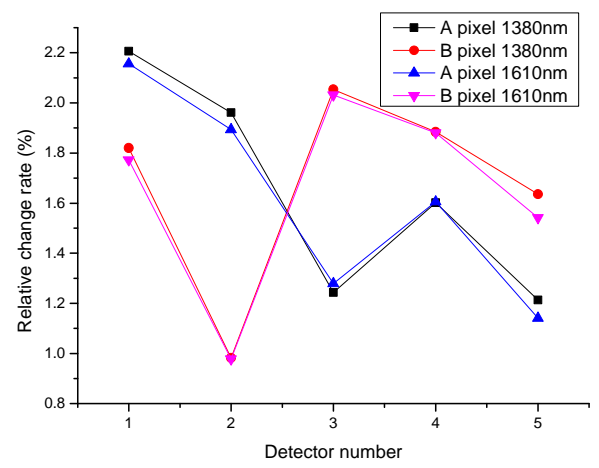

(a) G12180 detector
探测器作为一种典型的光电二极管, 其暗电流是 指在没有输入光信号的情况下输出的电流信号。探测 器暗电流来源复杂影响因素较多, 且其测量不确定度 也是影响短波红外偏振测量仪器测量精度的最重要 因素, 所以对于星载探测器组件进行试验前后暗电流 变化情况分析就显得尤为重要。图 7、图 8、图 9 分 别为各型号探测器试验前后暗电流值。

由图 7、图 8 可以看出: S13735 和 G12180 探测 器试验前后暗电流都不超过 $50 \mathrm{pA}$, 小于 $100 \mathrm{pA}$ 的要 求, 说明这几种型号的探测器组件经过试验考核后具 有较好的稳定性和可靠性, 满足航天载荷性能需求。 由图 9 可以看出: 对于 $\mathrm{G} 12183$ 探测器的 $0 \mathrm{mV}$ 偏压 和 $30 \mathrm{mV}$ 偏压也满足试验后暗电流变化率不超过试 验前 5 倍的要求, 同样满足航天载荷性能要求。

\section{3 制冷器制冷驱动电流试验前后变化}

红外探测器制冷器制冷驱动电流在室温 $22^{\circ} \mathrm{C}$ 条 件下进行测试(注: 编号 1 5 表示 5 只 InGaAs G12180 探测器, 编号 6 9 表示 4 只 InGaAs G12183 探测器), 测试结果及变化率如图 10 所示。

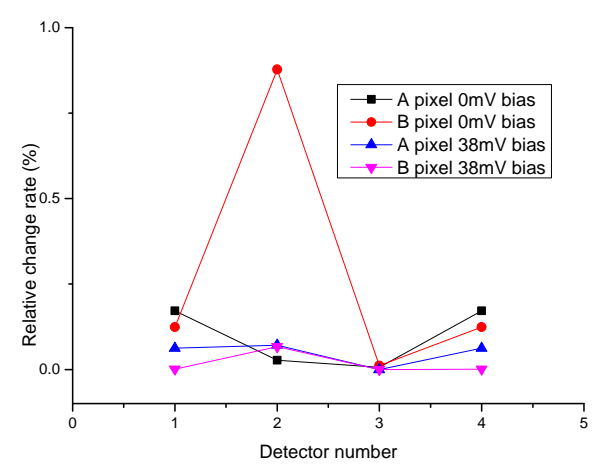

(b) G12183 detector

图 6 G12180 和 G12183 试验前后光谱响应率相对变化率

Fig.6 Relative change rate of spectral response before and after G12180 and G12183 tests

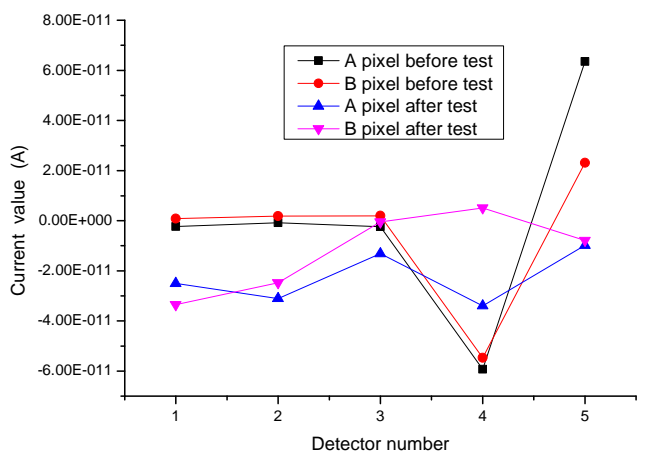

图 7 S13735 探测器试验前后暗电流值

Fig.7 Dark current before and after S13735 detector test

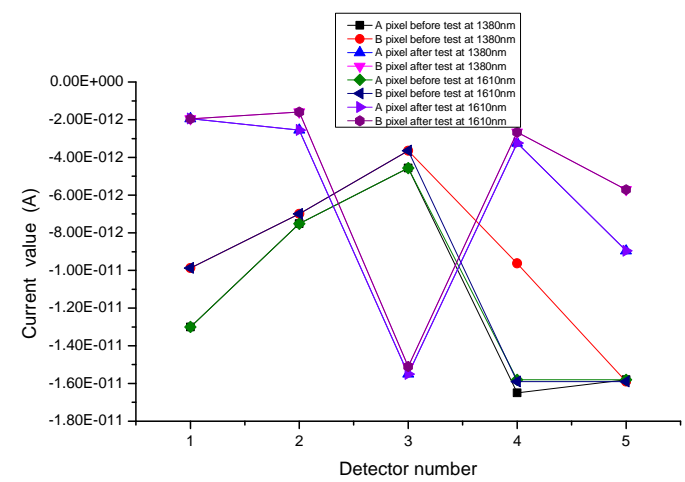

图 8 G12180 探测器试验前后暗电流值

Fig.8 Dark current value of G12180 detector before and after test 


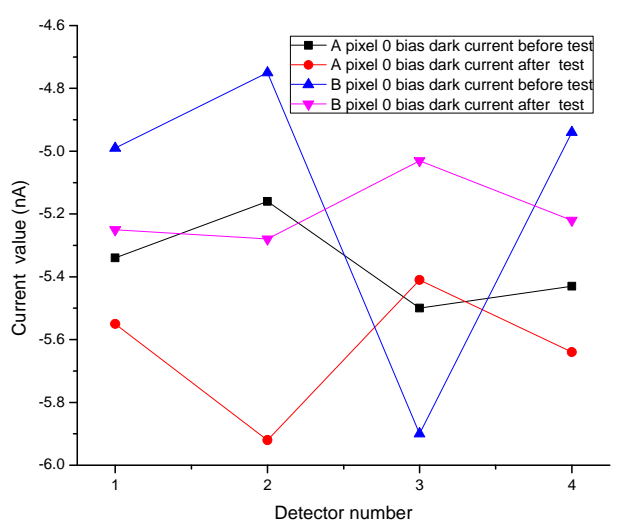

(a) Dark current value before and after $0 \mathrm{mV}$ bias test

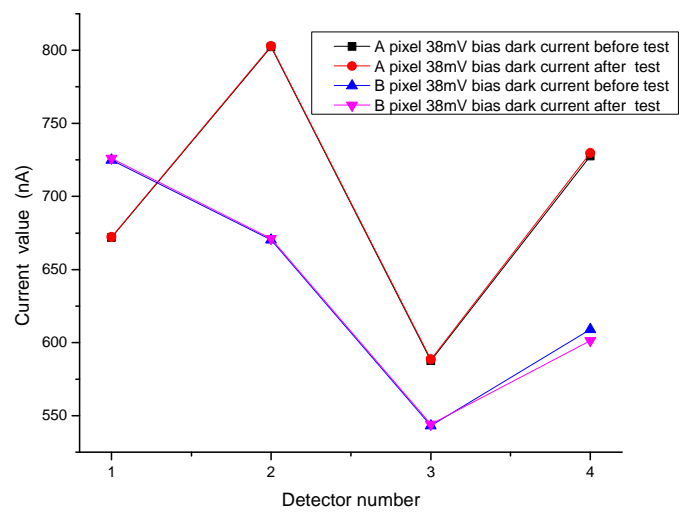

(b) Dark current value before and after $38 \mathrm{mV}$ bias test

图 $9 \mathrm{G} 12183$ 探测器 $0 \mathrm{mV}$ 和 $38 \mathrm{mV}$ 偏压试验前后暗电流值

Fig.9 Dark current value of G12183 detector before and after $0 \mathrm{mV}$ and $38 \mathrm{mV}$ bias test

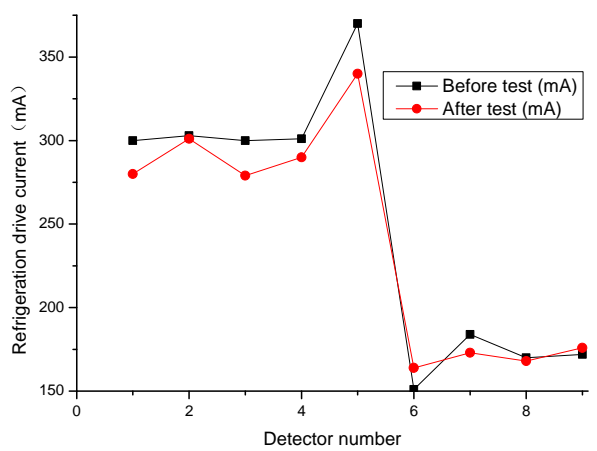

(a) Refrigeration driving current value

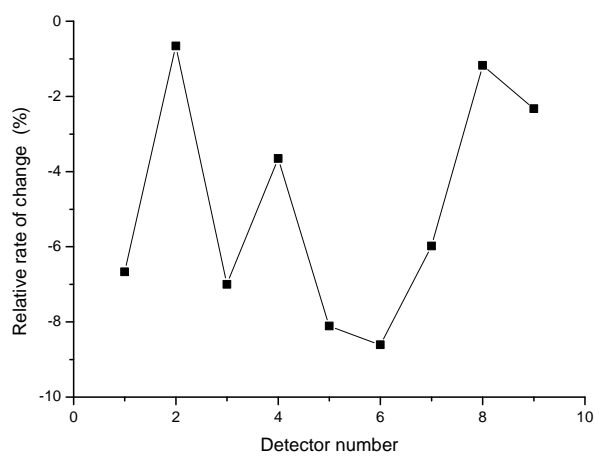

(b) Change rate of refrigeration driving current

图 10 红外探测器试验前后制冷驱动电流值及其变化率

Fig.10 Driving current value and change rate of refrigeration before and after infrared detector test

红外 InGaAs 探测器的 G12180、G12183 两种型 探测器试验前后的制冷驱动电流的变化率最大分别 为 $-8.108 \%$ 、 $-8.609 \%$, 也满足实验前初始测试值的 $\pm 10 \%$ 误差变化。

\section{4 探测器制冷特性测试结果}

红外 InGaAs 探测器制冷特性在探测器外壳温度 为 $-7^{\circ} \mathrm{C} \sim-17^{\circ} \mathrm{C}$ 时进行探测器制冷性能测试, 各个 探测器温控最大最小值测试结果如图 11 所示。

试验过程中, 红外探测器温控精度均满足 21.36 $\pm 0.2^{\circ} \mathrm{C}$ 要求, 探测器制冷特性满足要求。

\section{4 结论}

试验结果表明：S13735、G12180、G12183 型探 测器组件试验前后的相对光谱响应率最大变化率分 别为 $-1.3315 \% 、 2.2061 \% 、 0.8775 \%$, 满足试验前初 始测试值的 $\pm 10 \%$ 误差变化。S13735、G12180 型探测 器组件的试验前后的暗电流都不超过 $100 \mathrm{~Pa}, \mathrm{G} 12183$ 探测器的试验后暗电流变化率不超过试验前 5 倍, 皆
满足试验误差要求。红外 G12180 和 G12183 型探测 器的试验前后制冷器制冷驱动电流的变化率最大分 别为 $-8.108 \% 、-8.609 \%$, 满足实验前初始测试值的 $\pm 10 \%$ 误差变化, 探测器的制冷性能满足要求。

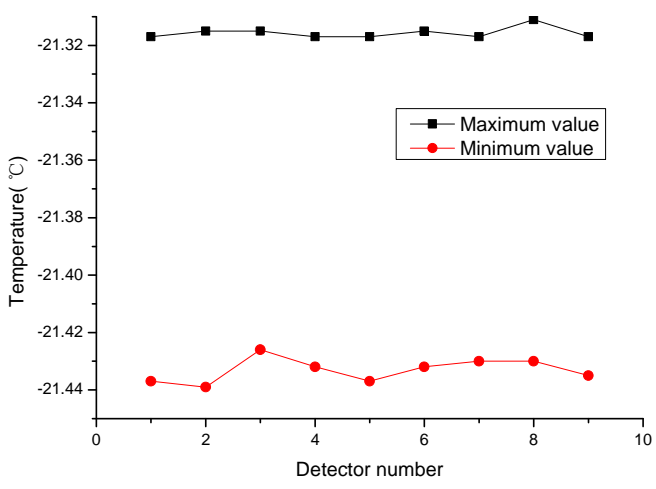

图 11 红外探测器温控值

Fig.11 Temperature control value of infrared detector assembly

红外探测器温控精度均满足 $21.36{ }^{\circ} \mathrm{C} \pm 0.2{ }^{\circ} \mathrm{C}$ 要 求。说明经过热真空环境试验考核后的各探测器组件 
具有较好的稳定性和可靠性, 探测器热真空试验后各 项性能指标正常。

结合各参数在实际工程中对探测器性能的影响 的重要性设置各参数权重因子, 进行定量计算后, 从 参试各类型探测器中选取试验综合性能最优的产品 用于星载高精度偏振和光谱测量中。

\section{参考文献:}

[1] 王炜鹏. InGaAs 光电探测器的可靠性研究[D]. 贵州: 贵州大学, 2019. WANG Weipeng. Reliability study of InGaAs photodetectors[D]. Guizhou: Guizhou University, 2019.

[2] 胡亚东, 胡巧云, 孙斌, 等. 暗电流对短波红外偏振测量精度的影响 [J]. 红外与激光工程, 2015, 44(8): 2375-2381.

HU Yadong, HU Qiaoyun, SUN Bin, et al. Influence of dark current on short wave infrared polarization measurement accuracy[J]. Infrared and laser engineering, 2015, 44(8): 2375-2381.

[3] 朱宪亮, 张海燕, 龚海梅. 航天 InGaAs 短波红外探测器步进应力加速 寿命试验研究[J]. 半导体光电, 2014, 35(6): 1008.

ZHU Xianliang, ZHANG Haiyan, GONG Haimei. Step stress accelerated life test of aerospace InGaAs short wave infrared detector[J]. Semiconductor Optoelectronics, 2014, 35(6): 1008.

[4] 洪津, 王征云, 胡亚东, 等. 星载红外探测器组件寿命试验研究及系统 设计 $[\mathrm{J}]$. 光学精密工程, 2018, 26(5): 1148-1155.

HONG Jin, WANG Zhengyun, HU Yadong, et al. Life test and system design of spaceborne infrared detector module[J]. Optical Precision Engineering, 2018, 26(5): 1148-1155.
[5] 任仁, 吴礼刚, 王小坤, 等. 碲镉永红外探测器高低温循环特性研究 [J]. 激光与红外, 2007, 37(S): 941-943.

REN Ren, WU Ligang, WANG Xiaokun, et al. Study on high and low temperature cycle characteristics of HgCdTe infrared detector[J]. Laser and Infrared, 2007, 37(s): 941-943.

[6] 朱双双, 吴洋, 邹鹏, 等. 星载红外探测器组件环境适应性分析与性 能优选[J]. 红外与激光工程, 2020, 49(2): 020400.

ZHU Shuangshuang, WU Yang, ZOU Peng, et al. Environmental adaptability analysis and performance optimization of spaceborne infrared detector module[J]. Infrared and Laser Engineering, 2020, 49(2): 020400 .

[7] 金伟, 陈迪虎, 李志伟, 等. 星载空间外差光谱仪探测器笁选测试方 法[J]. 中国激光, 2016, 43(9): 0904004.

JIN Wei, CHEN Dihu, LI Zhiwei, et al. Detector screening test method for spaceborne space heterodyne spectrometer[J]. China Laser, 2016, 43(9): 0904004.

[8] 李宪圣, 万志, 刘则洵, 等. 大视场空间相 CCD 性能测试及笁选方法 [J]. 红外与激光工程, 2014, 43(7): 2245-2250.

LI Xiansheng, WAN Zhi, LIU zexun, et al. Performance test and screening method of large field spatial phase CCD[J]. Infrared and Laser Engineering, 2014, 43(7): 2245-2250.

[9] 麦祖荫. 浴盆曲线及系统可靠性的数学理论[J]. 医疗装备, 1995(6): $1-2$.

MAI Zuyin. Mathematical theory of bathtub curve and system reliability [J]. Medical Equipment, 1995(6): 1-2. 